\title{
Los procesos de transformación territorial del área hortícola próxima a la ciudad de Bahía Blanca abordados a partir de talleres participativos con alumnos de las escuelas rurales y su aporte al desarrollo local
}

Os processos de transformação territorial área hortícola próximo à cidade de Bahía Blanca dirigida de oficinas participativas com os alunos de escolas rurais e a sua contribuição para o desenvolvimento local

The processes of territorial transformation of the horticultural area next the city of Bahia Blanca approached from participative workshops with students of the rural schools and their contribution to the local development

Les processus de transformation territoriale de la zone horticole proche de la ville de Bahía Blanca abordés à partir d'ateliers participatifs avec des élèves des écoles rurales et sa contribution au développement local

\author{
Laura de la Fuente* \\ (laura.delafuente@uns.edu.ar) \\ María Amalia Lorda** \\ (mariaamalial@yahoo.com.ar)
}

Recebido em 15/05/2015, revisado e aprovado em 23/07/2015; aceito em 22/11/2015 DOI: http://dx.doi.org/10.20435/1518-70122016105

\begin{abstract}
Resúmen: Las escuelas rurales han sido promotoras del desarrollo local del territorio por estar insertas y ser parte del mismo. El objetivo del presente trabajo es a través de los talleres, captar el imaginario social de los alumnos de su territorio hortícola. Los talleres se llevaron a la práctica en las escuelas rurales a través de la co-producción, talleres de reflexión sobre diversidad cultural, prácticas productivas y sociales en el medio rural.

Palabras clave: Escuelas rurales. Cartografía social. Desarrollo local.

Resumo: As escolas rurais foram as promotoras do desenvolvimento local do território para ser você insira e fazer parte do mesmo. O objetivo do trabalho presente é pelas lojas, capturar a uma reunião social imaginária dos estudantes de seu território hortícola. As lojas foram levadas à prática nas escolas rurais pela co-produção, lojas de reflexão têm diversidade produtiva e social cultural, prática mais que suficiente no meio rural.

Palavras chave: Escolas rurais. Cartografia social. Desenvolvimento local.

Abstract: Rural schools have been promoting local development of the territory by be embedded and be part of the same. The objective of the present work is through workshops, capture the social imaginary of the students of its horticultural territory. The workshops were to practice in rural schools through the co-production, reflection workshops on cultural diversity, social and productive practices in the rural environment.

Key words: Rural schools. Social cartography. Local development.

Résumé: Les écoles rurales ont été promotrices du développement local du territoire pour être ancrées et faire partie du même. L'objectif du présent travail est à travers des ateliers, attirer l'imaginaire social des élèves de son territoire horticole. Les ateliers ont été mis en pratique dans les écoles rurales grâce à la co-production, des ateliers de réflexion sur la diversité culturelle, pratiques productives et sociales en milieu rural.

Mots-clés: Écoles rurales. Cartographie sociale. Développement local.
\end{abstract}

\section{Introducción}

Numerosas son las obras literarias que hablan de la escuela rural y muestran la realidad de los pequeños poblados y de su gente, de sus esfuerzos por permanecer a pesar de las adversidades en el lugar, muchas de ellas promoviendo el arraigo. La escuela es un lugar donde los niños encuentran los pilares que fortalecerán su educación, donde se promueve en numerosos casos la contención, cuidados, espacios para el desarrollo del juego y apoyo a las familias rurales, entre otras cosas.

Considerando el contexto anteriormente descripto, se considera a la escuela rural como una institución que está inserta en un territorio, en la cual se observan una red de

\footnotetext{
* Universidad Nacional del Sur, Bahía Blanca, Argentina.

** Universidad Nacional del Sur, Bahía Blanca, Argentina. Laboratorio AGRITERRIS.
} 
relaciones con actores sociales diversos, que interactúan permanentemente y podría ser una entrada singular para comprender su forma de vida.

El trabajo de investigación abordado pone de manifiesto que la institución educativa rural es un actor de suma importancia en la construcción del desarrollo local del territorio, es un motor dinamizador de los procesos de desarrollo que allí ocurren, tanto sociales, económicos como culturales, entre otros. A partir del trabajo en el terreno desarrollado durante 15 años en el sector, es que se puede afirmar que la escuela en el medio rural, particularmente en este área hortícola, fue y sigue siendo parte clave en los procesos de migración.

\section{Caracterización del área productiva}

El área hortícola, en la cual se desarrolla esta investigación, comprende tres partidos del suroeste bonaerense, debido a que la zona productiva recorre los márgenes del rio Sauce Chico. Ellos son los partidos de Bahía Blanca, Villarino y Tornquist.

La actividad hortícola creció junto con la ciudad de Bahía Blanca y fue creando y construyendo el espacio inmediato, por ello podemos decir que su origen se remonta a principios del siglo pasado, y se la reconoce con la aparición y asentamiento de los primeros inmigrantes italianos, luego españoles y portugueses, así como los descendientes de ambos.

El área de estudio comprende la localidad de General Daniel Cerri y el área hortícola de la cuenca inferior del río Sauce Chico. A partir de la denominación que adquiere en la cartografía de base, se distingue al norte de la ruta nacional $n^{\circ} 3$ el área de Villa Elisa que agrupa a los núcleos hortícolas de Sauce Chico, Colonia La Merced y Alférez de San Martín; al sur de la ruta identificados como Villarino Viejo, y el sector de quintas de General Daniel Cerri (LORDA, 2005) y Nueva Roma accediendo por la ruta nacional $\mathrm{n}^{\circ} 35$, en el km 39,5.

\section{Los talleres como espacio de acción, producción y reflexión de los actores de un territorio}

Para captar el imaginario social del territorio hortícola, se llevó a la práctica en las escuelas rurales de nivel EGB (Escuela General Básica) a través de la co-producción, talleres de reflexión sobre diversidad cultural y prácticas productivas y sociales en el medio rural.

Estos talleres permiten reflexionar, considerándolos, no sólo como un dispositivo pedagógico, sino como una herramienta metodológica pertinente para este grupo de actores del territorio, como son los niños y docentes rurales.

Estos conceptos los refuerza la autora (GONZÁLEZ CUBERES, 1988) donde invita a pensar el taller como un espacio y un tiempo que se da para el aprendizaje, donde existe una transformación recíproca entre sujeto y objeto. En este espacio que brinda el taller aparece la acción, el sentimiento y el pensamiento de los participantes en plena libertad, junto a otros.

La autora define al taller de una manera muy expresiva y casi divertida, como lugar de "manufactura y mentefactura" (GONZÁLEZ CUBERES, 1987, p. 3), entendiendo mentefactura como la forma y la posibilidad de manifestar los saberes, los conocimientos. Sintetiza esta técnica como el lugar del vínculo, la comunicación y participación, donde existe una producción social de objetos, acciones y conocimientos de los participantes.

El taller propone una manera específica de intervenir, recolectar datos y construir o producir conocimiento; a partir de las diversas dinámicas que pueden incorporarse en él. Se interpela a todos los sujetos que participan. En este sentido, genera un espacio de acción, reflexión y producción colectiva de significaciones, en el que van emergiendo los sentidos que los habitantes de la comunidad le asignan a sus prácticas sociales.

La propuesta de trabajar en las escuelas rurales a través del Taller es un resultado de este proceso de reflexión en el que - como equipo junto a mi directora - se desarrolla para comenzar a entender que la mayoría de las referencias realizadas por cada individuo, sobre el espacio vivido y sentido por 
las familias, se encuentra sujeto a un sistema de relaciones, costumbres y creencias hilvanadas en el tejido social y familiar que brinda el establecimiento educativo. Éstos, a su vez, delinean el conjunto de presupuestos, sentidos y prácticas, produciendo disputas y consensos hacia el interior de la comunidad y definiendo su identidad.

En este sentido, el equipo planifica su intervención a través del taller, como en el caso de cualquier estrategia metodológica. Éste permite incorporar las inquietudes y necesidades del espacio social e institucional en el que es llevado a cabo, como un proceso abierto, principal motivo por el cual se lleva a la práctica.

Es importante resaltar que es fundamental plantear instancias de trabajo con las instituciones en su propio ámbito, en lugar de convocarlos a un espacio diferente. El hecho de desarrollar las actividades en el propio espacio de la institución, con su sentido e incertidumbres, está íntimamente relacionado con el interés por investigar actuando.

Otra forma de investigar durante la realización de los talleres con los niños, es a través del método de observación participante, sobre todo en los momentos en que los alumnos realizan sus producciones gráficas y en el trabajo grupal propiamente dicho.

La observación participante permite encontrar el despliegue de relaciones e interacciones "cara a cara" compartiendo con los actores, en este caso, alumnos y docentes, actividades y sentimientos durante un determinado periodo de tiempo, constituyendo un soporte fundamental para el trabajo de investigación (AMEIGEIRAS, EN VASILACHIS S/A).

En los primeros momentos de la observación, predomina una mirada general y amplia, para luego focalizar más profundamente en los actores, situaciones y procesos que ocurren durante el desarrollo del taller, en este caso, en particular.

El taller hace posible desarrollar un postulado de la investigación-acción que comprende a la misma como un proceso educativo. Y se define como la liberación del potencial creador y la movilización de los recursos humanos para la solución de los problemas sociales y con ésta la transformación de la realidad social (SCHUTTER, 1983). Desde este encuadre, el taller constituye un espacio de acción, reflexión y producción colectiva de significaciones, dado que todas las dinámicas que se proponen en este marco interpelan a todos los sujetos que participan de él. Por esto, también es entendido como una experiencia pedagógica a partir de un método en el que se aprende mientras se realiza una actividad. Pero en el que, además se debe lograr generar un ambiente de armonía, libertad y confianza en sí mismo y en los demás.

Esto último genera las condiciones apropiadas para descubrir y analizar el imaginario social de las diferentes comunidades educativas, a través de una interrelación colectiva. Es decir, todos ubican en ese espacio las subjetividades y en el hacer van surgiendo los diversos significados que cada individuo le asigna a las cosas.

Según palabras de Raúl Ageno (1989, s/n); por su dinámica de funcionamiento, el taller: "requiere un ámbito de cercanía afectiva, un telé positivo que permita/posibilite una comunicación fluida para que la palabra circule con libertad. Esta situación, sumada a la práctica en la cual son entrenados los alumnos trabajando su propio saber en conjunto con los transmitidos por los docentes, favorece la construcción de conocimientos en forma grupal, reflexiva e inteligente y posibilita el tránsito del saber del sentido común al saber científico".

\section{La cartografía social: una propuesta} metodológica que permite profundizar el territorio socialmente construido a partir del taller realizado en las escuelas rurales

Una parte importante de esta investigación se realiza en las instituciones educativas, donde se decide implementar la modalidad de taller y a través del mismo aplicar la propuesta metodológica de la cartografía social con los niños que asisten a las mismas. Diferentes edades componen el grupo de trabajo de los niños; participan junto con ellos, docentes e investigadoras de la universidad ${ }^{1}$ con diferentes competencias. Estos establecimientos educativos, al tener una escasa matricula, funcionan integradamente, es decir, las salas

\footnotetext{
${ }^{1}$ La animación de los talleres la realizan las autoras del presente trabajo.
} 
o grados abarcan las edades desde los 6 años hasta los 12 años inclusive.

Es interesante abordar el concepto de cartografía social. En primer lugar, se rescata la definición que la Asociación Cartográfica Internacional brinda para el vocablo mapa: "Es la representación convencional grafica de fenómenos concretos o abstractos, localizados en la Tierra o en cualquier parte del Universo" (1959).

En los mapas se encuentran conjunto de signos, dibujos muy simples, como un croquis dibujado en papel, y otros más complejos, impresos en muchos colores. Pero se puede afirmar que un mapa es siempre el esquema de la realidad.

¿Por qué elegir y/o tomar en consideración esta propuesta metodológica? En todas las comunidades, el planeamiento, los proyectos, la participación, compromiso y los sueños de las personas que lo habitan, no se hacen en el aire, se desarrollan y se viven intensamente en un territorio determinado.

Dicho territorio es un espacio cultural, donde se interrelacionan el hombre y la naturaleza, con diversidad y multiplicidad de etnias, como es el caso de este terreno de investigación. Allí las culturas, los conocimientos, saberes y practicas están en continua relación y transformación por parte de los actores de esa comunidad (CARBAJAL, 2005).

En este caso la cartografía social, juega un papel muy importante, se la considera para este estudio como una herramienta metodológica de gran ayuda, particularmente para el análisis y comprensión del territorio, de "su territorio". Permite a través de su utilización un acercamiento a la comunidad a su espacio geográfico, social, económico e histórico-cultural. En palabras de Diez Tetamanti e Escudero (2012, p. 10) "jugamos seriamente a recordar, debatir y soñar nuestro territorio con una única proyección: la colectiva".

La cartografía social utilizada en esta investigación, a través de los talleres con los alumnos, permite indagar en el sentido de reafirmación de la pertenencia a un territorio. A partir de esta herramienta utilizada adecuadamente, se puede conocer, por medio del dibujo, una realidad, generar un espacio de encuentro de los diferentes actores sociales de una comunidad (alumnos, maestros, directores, investigadores), así como reforzar el sentido de pertenencia al reconocer el territorio e ir descubriendo cómo construyen su identidad (CARBAJAL, 2005).

\section{La cartografía social y su relación con la investigación-acción-participativa (IAP)}

La cartografía social tiene como fundamentos conceptuales la investigación- acción-participativa (IAP). Es importante especificar algunas conceptualizaciones sobre esta metodología:

"La investigación-acción es una actividad integrada que combina la investigación social, el trabajo educacional y la acción" (HALL, 1981). Según ANDER-EGG (1990, s/n), "La investigación - acción - participativa supone la simultaneidad del proceso de conocer y de intervenir e implica la participación de la misma gente involucrada en el programa de estudio y acción".

Desde este encuadre, resulta importante aclarar que en la" investigación", todos los miembros que participan de la comunidad objeto de estudio, son parte de la investigación, ya que aportan sus conocimientos, saberes implícitos y experiencias cotidianas, al mismo tiempo que recibe en forma consciente e inconsciente de los demás. En esta investigación el elemento fundamental que subyace en la cartografía es el territorio, sobre el que se abordan los tres elementos que fundamentan la IAP, y sobre los que se va a detallar en los párrafos sucesivos. La construcción colectiva de mapas permite recuperar la memoria individual y colectiva de los actores involucrados.

Respecto a la "acción" al conocer y/o descubrir la realidad se puede actuar sobre ella, es decir permite transformarla y no sólo investigar para conocerla. Se pretende que la acción conduzca a la construcción social del territorio, a nuevos lazos que permitan fortalecer un entramado social signado por cierta vulnerabilidad.

La "participación" es un proceso continuo de construcción social, que tiene que ver con los aportes del conocimiento, las experiencias vividas y propuestas de transformaciones para el desarrollo del lugar que habitan. Es necesario que la participación sea activa, comprometida, organizada, respetuosa, que conlleve a la toma de decisiones. En 
cada proceso de gestión es imprescindible la participación de la comunidad.

A su vez, la "sistematización" implica no sólo organizar la información, sino sobre todo descubrir la coherencia interna de los procesos instalados en la práctica. Es como traducir en teoría la práctica vivida durante ese período de trabajo conjunto. La sistematización permite al investigador y a la comunidad dimensionar los conocimientos, los datos y las prácticas para hacer posible el desarrollo social de la comunidad en el territorio.

La cartografía social es considerada una nueva herramienta de planificación y transformación social, fundamentada en la investigación - acción - participativa y sus principios. Esta herramienta constituye un aporte a la planificación participativa, organización de redes, formación de ciudadanía, entre otros.

Se puede afirmar que su puesta en práctica favorece la construcción de un conocimiento integral de un territorio, partiendo desde la participación y el compromiso social, dando la posibilidad de transformar al mismo, a partir de la detección de percepciones comunes o no, pero que alimentan una nueva concepción de su territorio. Este instrumento permite rescatar el conocimiento colectivo, acercar la comunidad a su espacio de vida, a su espacio socio-económico, histórico y cultural (HABEGGER; MANCILA, 2006).

Se logra construir el conocimiento a través de la elaboración por parte de los individuos de mapas, a manera de simples dibujos, mediante el intercambio grupal de sus vivencias, sentimientos y percepciones. Esto permite que se desarrollen procesos de comunicación entre los participantes y se facilita a través del mismo, observar los diferentes tipos de saberes que aparecen, que se entrelazan, dando de ese modo, una imagen colectiva del territorio.

Es interesante destacar que es posible elaborar numerosos tipos de mapas: mapas del pasado, presente y futuro, como también "mapas temáticos" que ofrecen un mayor conocimiento del espacio donde habitan, tales como mapas administrativo; infraestructurales; económicos; mapa ecológicos; mapa de redes de relaciones; de conflictos; entre otros.

A continuación, se detalla la información que permite obtener cada uno de los diferentes tipos de mapa, así como también la finalidad que se persigue:

- Mapa del pasado: permite reconocer cambios del contexto y permite a su vez rescatar la memoria colectiva de sus habitantes.

- Mapa del presente: da una idea de la situación actual del terreno, del espacio en que se habita. Se puede observar la realidad inmediata.

- Mapa del futuro: se los puede definir también como "mapa de los sueños", en el se puede visualizar cómo quieren que sea su barrio, su colonia, su quinta, su espacio donde vive, para el día de mañana.

En esta oportunidad el mapa, y particularmente el mapa del presente, es el centro de inspiración de los participantes, que se emplea mediante talleres, grupos de discusión, permitiendo de ese modo el redescubrimiento del territorio pudiendo hablar sobre el mismo y las territorialidades.

Además del mapeo se pueden utilizar otros instrumentos en forma complementaria como pueden ser, la entrevista, la observación participante, a los que que también son importantes de considerar instrumentos del tipo vivencial como por ejemplo: recorridos de campo con las personas de la comunidad, juegos, narración de experiencias cotidianas, creaciones simbólicas del lugar, material visual

En el caso del terreno que se aborda en esta investigación, a través de la cartografía social, se puede conocer por medio del dibujo que los niños han elaborado en la escuela, una realidad; su realidad. Ofrece, además, un nuevo espacio de encuentro de los diferentes actores sociales de la comunidad, y permite reforzar el sentido de pertenencia al reconocer su lugar, su espacio y de ese modo, reafirmar su identidad y su cultura.

\section{La experiencia práctica de la cartografía social en talleres}

La propuesta de trabajo surge en primera instancia a través de un proyecto de Extensión Universitaria, que presenta el Departamento de Agronomía, donde aborda la problemática hortícola local, tanto en aspectos económicos, comerciales, sociales (salud, alimentación) y de gestión de las pequeñas familias rurales. Es allí donde 
aparece la propuesta de trabajar en equipo interdisciplinario, docentes del Departamento de Geografía y Turismo junto con docentes del Departamento de Agronomía. Surge así como propuesta trabajar con la modalidad de talleres, con alumnos de las escuelas rurales en temas referidos a la alimentación saludable, el consumo de hortalizas, la valoración del espacio de la producción primaria y valoración del trabajo de las familias en la quinta.

Se abordaron los talleres en dos escuelas rurales, del área en estudio. Se trabajaron en variadas temáticas, como fueron la alimen- tación saludable, el consumo de hortalizas, la valoración del espacio de la producción primaria y valoración del trabajo de las familias en la quinta con el objeto de descubrir la importancia que le dan a la alimentación en sus hogares, acompañarlos en un proceso de valoración de la actividad hortícola de sus familias, como fuente primaria de alimentación, entre otros.

El encuentro, en si llevaba el nombre "Que lindo es mi lugar y qué importante los alimentos que allí se producen".

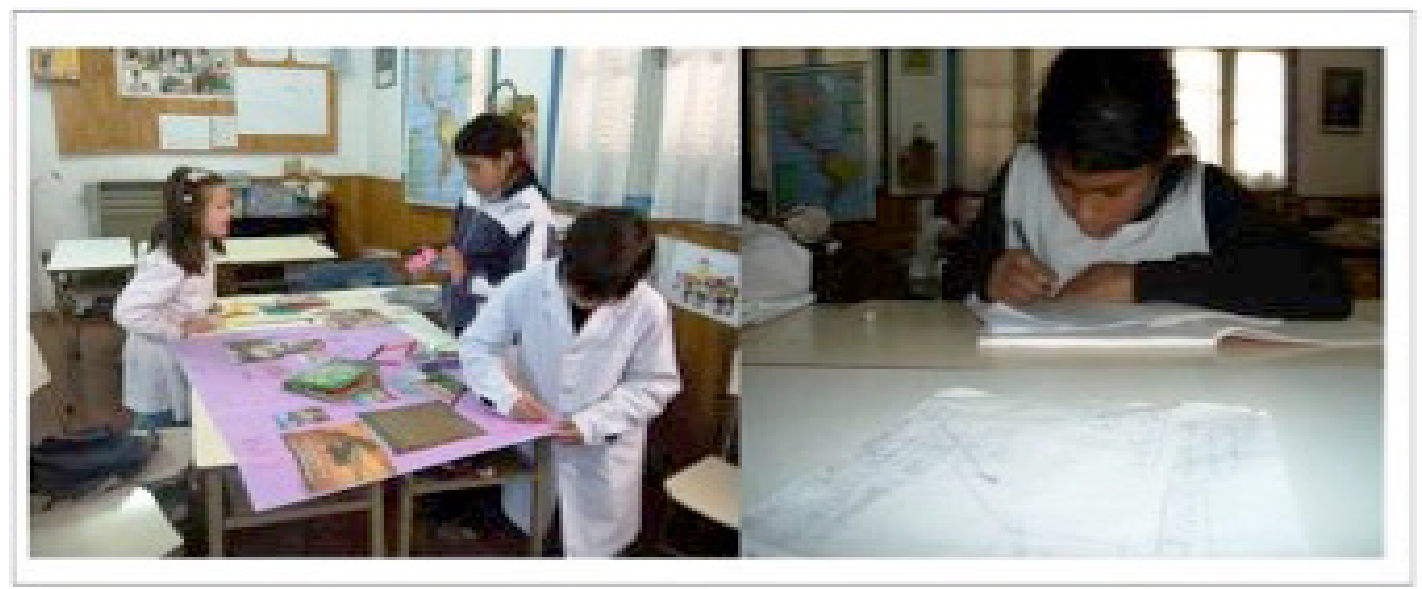

Fotos 1 y 2 - Alumnos trabajando en el diseño de los afiches

Las actividades realizadas respondían a diferentes objetivos planteados por las investigadoras, se trabaja con el croquis de la imagen mental, donde cada alumno destaca mediante dibujos sencillos cómo es el lugar que habitan.

Seguidamente, se propone a través de la técnica de lluvia de ideas, cuáles son las comidas favoritas, con la finalidad de conocer a través de esta técnica sus hábitos alimenticios, animando a los alumnos a su vez, a que descubran los diferentes grupos de alimentos y la importancia de cada uno de ellos en la alimentación.

A continuación, otra actividad propuesta, fue, a través de un sistema de preguntas simples redactadas en tarjetas, por las investigadoras. De ese modo se intentó conocer más sobre los alimentos y su lugar de vida. Luego de haberse aplicado la técnica, se hace una una puesta en común pegando y presentando sus tarjetas en el pizarrón. Ya acercándonos al final del taller, se presenta al grupo la nueva imagen de la pirámide nutricional, que anteriormente era la pirámide nutricional y en la actualidad es reemplazada por el "Ovalo nutricional". La finalidad de esta etapa es aprehender los nuevos conceptos de alimentación saludable y la importancia fundamental del agua en el proceso, así como del cuidado de los alimentos en toda la cadena de producción hasta llegar a la mesa.

Como actividad final se les propuso realizar en afiche, una propaganda, promocionando el lugar donde viven y lo que allí se produce con el objeto de dar a conocer el lugar donde habitan, la cercanía a la ciudad de Bahía Blanca y la importancia de la producción de las verduras que allí sus padres realizan, como así también dar visibilidad de este territorio a la población urbana, de la actividad hortícola y rural. 


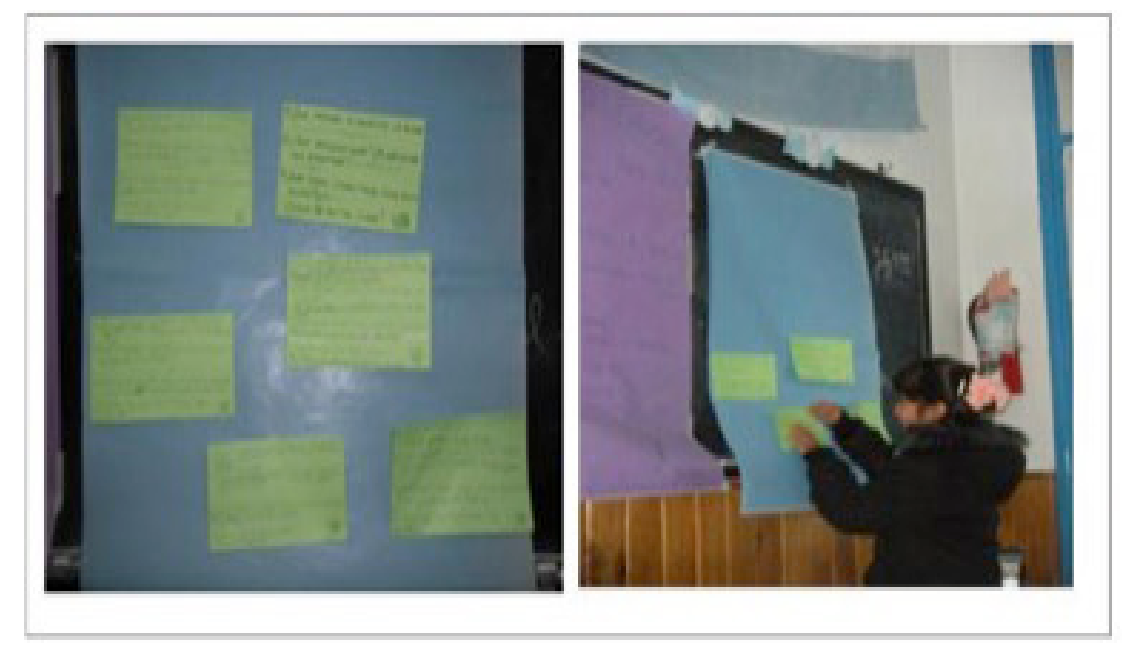

Fotos 3 y 4 - Puesta en común de la propuesta de los alumnos

Las actividades, a lo largo de los talleres, han sido desarrolladas en un ambiente de cordialidad y de sumo interés por parte de alumnos y docentes. El afecto manifiesto por los niños ha sido movilizante para las investigadoras, como así también la predisposición de la institución para llevar adelante los mismos. Las investigadoras han tenido la posibilidad de compartir un almuerzo con la comunidad en un ambiente muy distendido que hizo de esos encuentros, momentos muy placenteros.

\section{Reflexiones finales}

Los diferentes talleres llevados a cabo en las escuelas rurales han permitido considerar a la escuela rural como una institución que está inserta en un territorio, en la cual se observan una red de relaciones con actores sociales diversos, que interactúan permanentemente y podría ser una entrada singular para comprender su forma de vida.

El trabajo de investigación realizado pone de manifiesto que la institución educativa rural es un actor de suma importancia en la construcción del desarrollo local del territorio, es un motor dinamizador de los procesos de desarrollo que allí ocurren, tanto sociales, económicos como culturales, entre otros. A partir del trabajo en el terreno desarrollado durante quince años en el sector, es que se puede afirmar que la escuela en el medio rural, particularmente en este área hortícola, fue y sigue siendo parte clave de los territorios.
A través de la aplicación de la cartografía social y en una dinámica grupal de taller se pueden analizar y comprender diferentes aspectos de la vida en la quinta, la alimentación de las familias y aspectos nutricionales, como así también, la imagen que tienen de su lugar de vida, sus inquietudes y necesidades, el reconocimiento del paisaje que habitan, la importancia que le dan al aire libre, a la naturaleza, entre otros.

Esta metodología, permite a través de su utilización un acercamiento a la comunidad, a su espacio geográfico, social, económico e histórico-cultural. Los alumnos de este modo han construido un mapa del presente, con sus representaciones simbólicas del lugar donde viven.

Analizando cada una de las actividades realizadas, se puede observar los aspectos favorables y desfavorables que posee, para ciertos alumnos, la vida en la quinta y en general en el medio rural.

A su vez Las respuestas de docentes y alumnos a la propuesta de trabajo llevada a cabo en los dos establecimientos, es favorable, a la vez alentadora y enriquecedora para la labor de investigación, tanto como el trabajo en docencia, en la Universidad, ámbito en el cual las investigadoras pertenecen.

En el marco del taller, las actividades se llevaron a cabo con entusiasmo y alegría, de una manera desestructurada, y así fueron vivenciadas por los niños con gran naturalidad y un ambiente de confianza donde pudieron expresarse con libertad y creatividad 


\section{Referencias}

AGENO, R. El Taller de Educadores y la Investigación. Cuadernos de Formación Docente, n. 4, Secretaria Académica, Universidad Nacional de Rosario, 1989.

ANDER-EGG E. Repensando la Investigación-Acción Participativa. [s.1.]: Editorial Lumen, 1990. 177p. (Colección Política servicios y trabajo social).

CARBAJAL, J. C. Territorio y cartografía social. Fortalecimiento de las organizaciones pertenecientes a la asociación de proyectos comunitarios. Popayan: Asociación de Proyectos Comunitarios - APC; Módulos de Trabajo y Estudio, 2005

CARTA topográfica de la República Argentina n $3963-17-1$. Provincia de Buenos Aires, Bahía Blanca: Instituto Geográfico Militar, 1969.

DIEZ TETAMANTI, J. M.; ESCUDERO, B. (Comp.). Cartografía social: investigaciones e intervención desde las ciencias sociales: métodos y experiencias de aplicación. 1. ed. Comodoro Rivadavia: Universitaria de la Patagonia, 2012.

GONZÁLEZ CUBERES, M. T. El taller de los talleres. Buenos Aires: Estrada, 1988.

HABEGGER, S.; MANCILA, I. El poder de la Cartografía Social en las prácticas contra hegemónicas o La Cartografía Social como estrategia para diagnosticar nuestro territorio. Revista Araciega, 14, 2006.

HALL, B. Investigación participativa, conocimiento popular y poder: una reflexión personal. México: Biblioteca Digital CREFAL, 1981.

LORDA, M. A. El desarrollo local, estrategia de gestión ambiental de la actividad agropecuaria en espacios próximos a la ciudad de Bahía Blanca. Tesis (Doctoral) - Departamento de Geografía y Turismo, UNS, Argentina, 2005.

SCHUTTER, Anton. Investigación participativa: una opción metodológica para la educación de adultos. México: CREFAL, 1983. 